Scientific Visualization, 2019, volume 11, number 2, pages 1 - 10, DOI: 10.26583/sv.11.2.01

\title{
Shadow scheme with selective range of photoregistration in aerodynamic tests
}

\author{
S.I. Gerasimov'1,A,B,C,D, V.A. Kikeev,a,D, V.A. Kuzmin3,A,B,C, \\ K.V. Totyshev4,C, A.P. Fomkin5,C, R.V. Gerasimova ${ }^{6, B}$ \\ A Institute of Problems of Mechanical Engineering, Nizhny Novgorod, Russia \\ в Sarov Physics and Technical Institute of NRNU «MEPHI», Russia, \\ Nizhny Novgorod region \\ C Russian Federal Nuclear Center - All-Russia Research Institute of Experimental Physics, \\ Russia, Sarov, Nizhny Novgorod region \\ D Nizhny Novgorod State Technical University n.a. R.E. Alekseev, Russia \\ ${ }^{1}$ ORCID: 0ooo-0002-6850-0816, s.i.gerasimov@mail.ru \\ ${ }^{2}$ ORCID: 0000-0002-2375-0803 \\ 3 ORCID: 0000-0002-7141-0408 \\ 4 ORCID: 0000-0002-6232-505X \\ 5 ORCID: 0000-0002-7787-7121 \\ 6 ORCID: 0000-0002-2711-3975
}

\begin{abstract}
$\underline{\text { Abstract }}$
The classical straight-cut registration scheme has a high resolution and the absence of distortions associated with the use of optics. The principal drawback that does not allow the use of this scheme in the field - susceptibility to background radiation accompanying the experiment. Number of recorded processes are characterized by relatively small size of the objects being thrown, for example, in the problem of modeling the impact of space "debris" with given screens or in the problem of modeling the emergency expansion of fragments of the active material. Resolution of x-rays is insufficient to render such particles, and their hypersonic speed and the corresponding ionization of the air result in the exposure of photographic film in an open straight-cut scheme. Additional background highlights in such experiments are the emission of muzzle exhaust in the case of use for dispersal of ballistic barrel systems or the glow of the explosion products in the case of detonation accelerating devices. Meanwhile, opportunities of straight-cut scheme can be expanded by spending experience in the twilight time of the day and protected from background radiation, for example, by raccord as a filter of large area. The paper describes a shadow scheme of photo registration, which includes a point light source and a wide-format film with a protective filter. The result of application of this scheme in aerodynamic tests accompanied by intense background illumination is presented.
\end{abstract}

Keywords: photographic, isopanchromatic film, filter, pulse light source. 


\section{Introduction}

An accident of products with explosives might lead to a fragmentation of fissile materials (FM) and formation of both small (less than 100 microns) and larger particles (fragments). During the expansion process, the interaction of high-speed particles with the products of the explosion (EP) causes their thermochemical destruction and the formation of smaller particle-fragments, which makes an additional contribution to the spectrum of fine particles. Experimental data on the spectrum of additionally formed particles during the interaction of FM and EP fragments is necessary to determine their contribution to the radioactive pollution of the district and to develop technical decisions how to reduce radiation consequences for similar accidents.

Experiments with the specimens (models) containing FM are expensive and pose the environmental hazard. Therefore, a methodology to study the dispersion of FM in emergency situations involving the explosion and simulating FM is developed, and it allows to determine the contribution of high-speed fragments of FM in the General spectrum distribution. The way to simulate the properties of plutonium using a simulator material (cerium metal) (which behaves similarly to FM when it is exposed to hypersonic airflow) is environmentally safe (patent RF № 2298850). To solve the problems of studying aerosols from FM simulators, techniques have been developed which allow throwing a group of solid parallelepiped shaped particles with the sizes from $1.4^{*} 0.8^{*} 0.9$ to $1.4^{*} 0.8^{*} 1.1 \mathrm{~mm}$ with hypersonic velocities and to carry out optical non-contact registration of this group and aerosols formed from the solid particles of this group in while flying. Initial experimental studies have shown that the flight of particles of the FM simulator in the air atmosphere with an initial velocity of $\sim 3000 \mathrm{~m} / \mathrm{s}$ causes thermomechanical particles destruction in the solid state with the formation of a large number of particles due to their oxidation during heating and braking in the incoming highenthalpy flow.

Thus, it has become necessary to develop an optical non-contact way to register these particles.

1. Shadow scheme with selective range of photorecording.

Pulsed light sources with gas-discharge and tube emitters are widely used in optical registration practice [1]. The main advantage of these light sources is that they have a high brightness temperature and a wide range of radiation with the maximum at ultraviolet.

Today, despite the active transition to digital technology, there are still tasks at which photographic materials are used. Isoperational film has photosensitivity most fully overlapping a visible emission spectrum. The maximum sensitivity remains at the ultraviolet region (Fig. 1). 


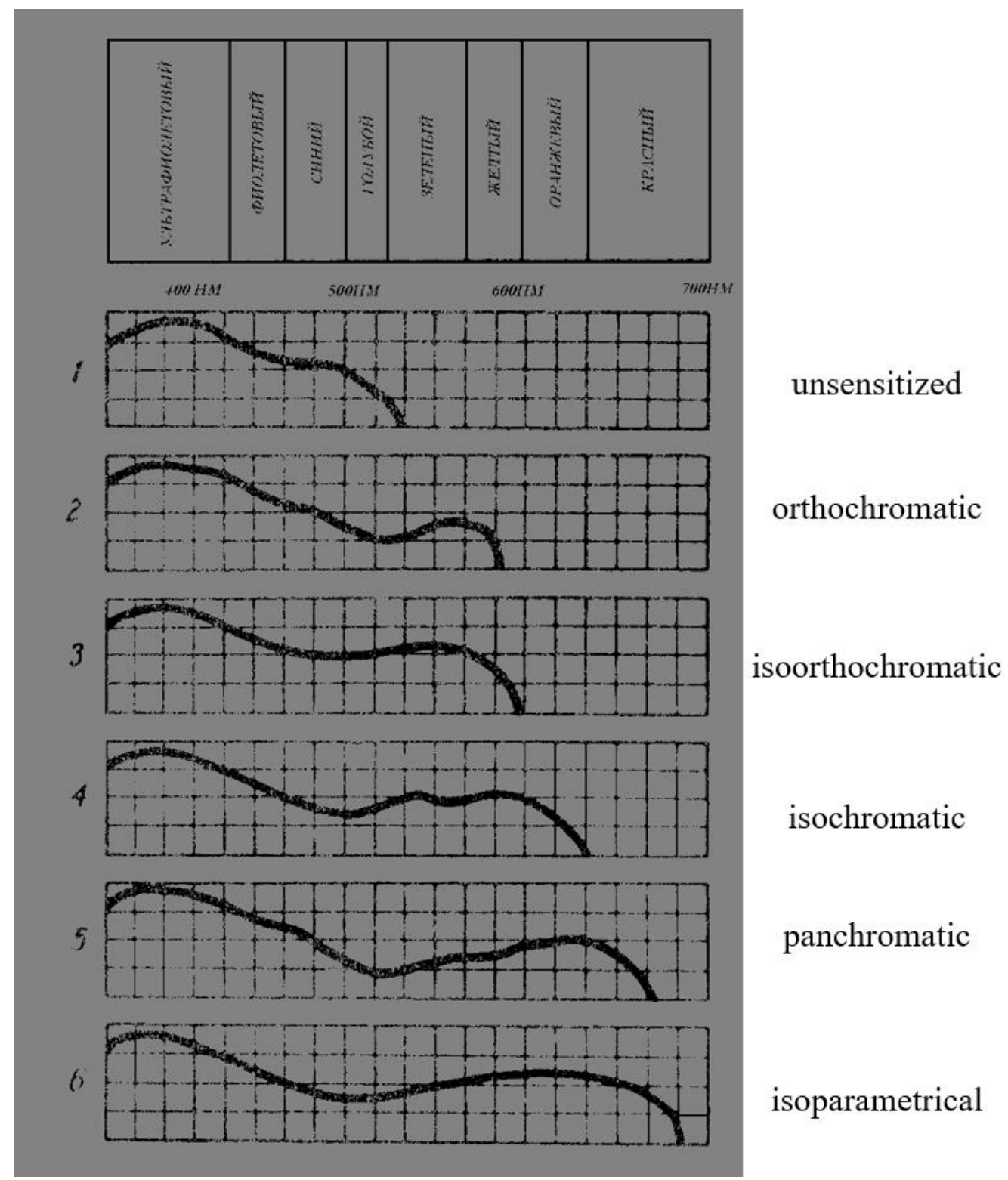

Fig. 1 - Photosensitivity curves for differently sensitized photographic materials

The classical scheme of a shadow image construction is a scheme where a point source of radiation (the size of the glow body is much smaller than the distance to the screen) builds a shadow picture from the test object (OI) in divergent rays on a photosensitive material (film, photo paper or photographic plate) [2-12]. At the same time, due to the dependence of the refractive index on the density, the angle of inclination also changes with the passage of optical inhomogeneities, which makes it possible to visualize, in particular, the head shock wave and the turbulent trace of the OI at supersonic speeds on the photographic material. The main drawback of the photographic material is that it is a single-use receiver. However, out of all image receivers, it is still the only one which has both high sensitivity and high resolution. Application of the classical shadow scheme in the ballistic experiment is not always possible due to the strong background radiation, for example, during the intensive process of hypersonic aerodynamic destruction or during the photo-recording of the intermediate ballistic trajectory of the intermediate ballistics [13,14]. Improvement of the basic and introduction of additional elements to the scheme of photo registration allows one to solve similar problems using the shadow method. The main elements of this scheme are the light source and the screen (photographic material), the set of their parameters determines the spatial resolution of the optical scheme. The sensitivity of the circuit increases proportionally to the distance from the source to the screen. The shadow picture is more distinct the smaller the light source 
and the closer the registration object is to the screen [1]. Since the use of an open film in the wording of the formulated problem is impossible, to protect the negative from intense background illumination on a cassette with an isopanchromatic film, a protective light filter is installed to cut off the background radiation in the green-red range (the spectrum of background radiation of combustion and explosion products). Glass filter SS-8 and membrane filter record have been considered. The distribution of spectral sensitivity of these filters was measured and is shown on Fig. 2.

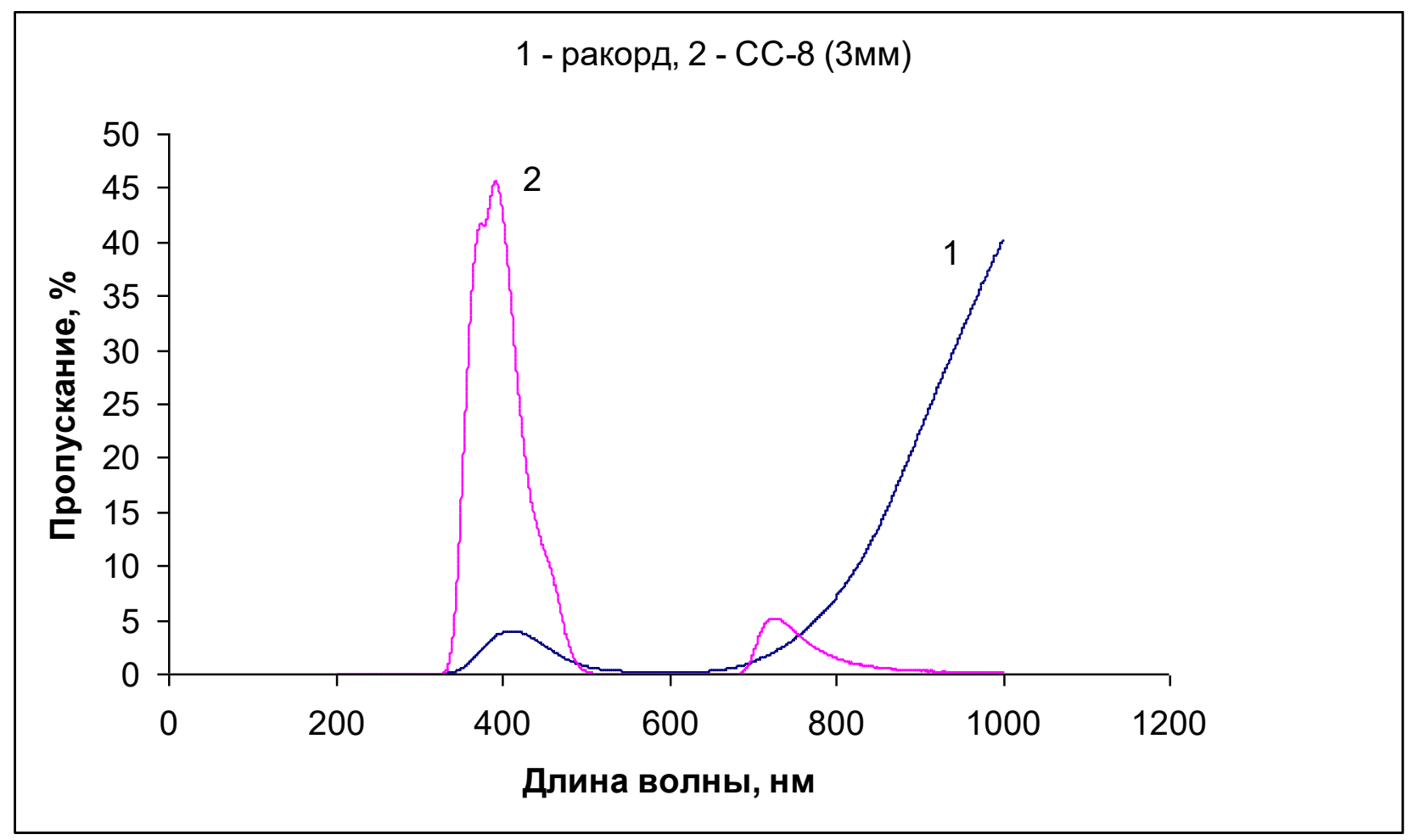

Fig. 2-diagram of the spectral sensitivity of the two filters.

Both filters have similar parameters, film area of $700 \mathrm{~nm}$ or more is not sensitive, and therefore is not of interest. Given the fact that the gas-discharge light sources are characterized by a brightness temperature of 10,000 to $30,000 \mathrm{~K}$ (ultraviolet-violet region), the use of both filters is optimal. The greatest transmittance in the range of $350 \div 500 \mathrm{~nm}$ has a glass filter SS-8, but in the field test conditions the film filter racord is more applicable. Thus, the shadow scheme (Fig. 3) consists of a point discharge source 1 with EVIS emitter [1] with aperture size of $7 \mathrm{~mm}$, which is located in front of the cassette 2 with a large open isoperational film 3 closed by a filter, "record" 5 . In addition, specifically for this scheme a light-tight mobile stand of shadow photo registration 4 with input and output windows for through movement of the object 6 in the registration area was designed and manufactured. 


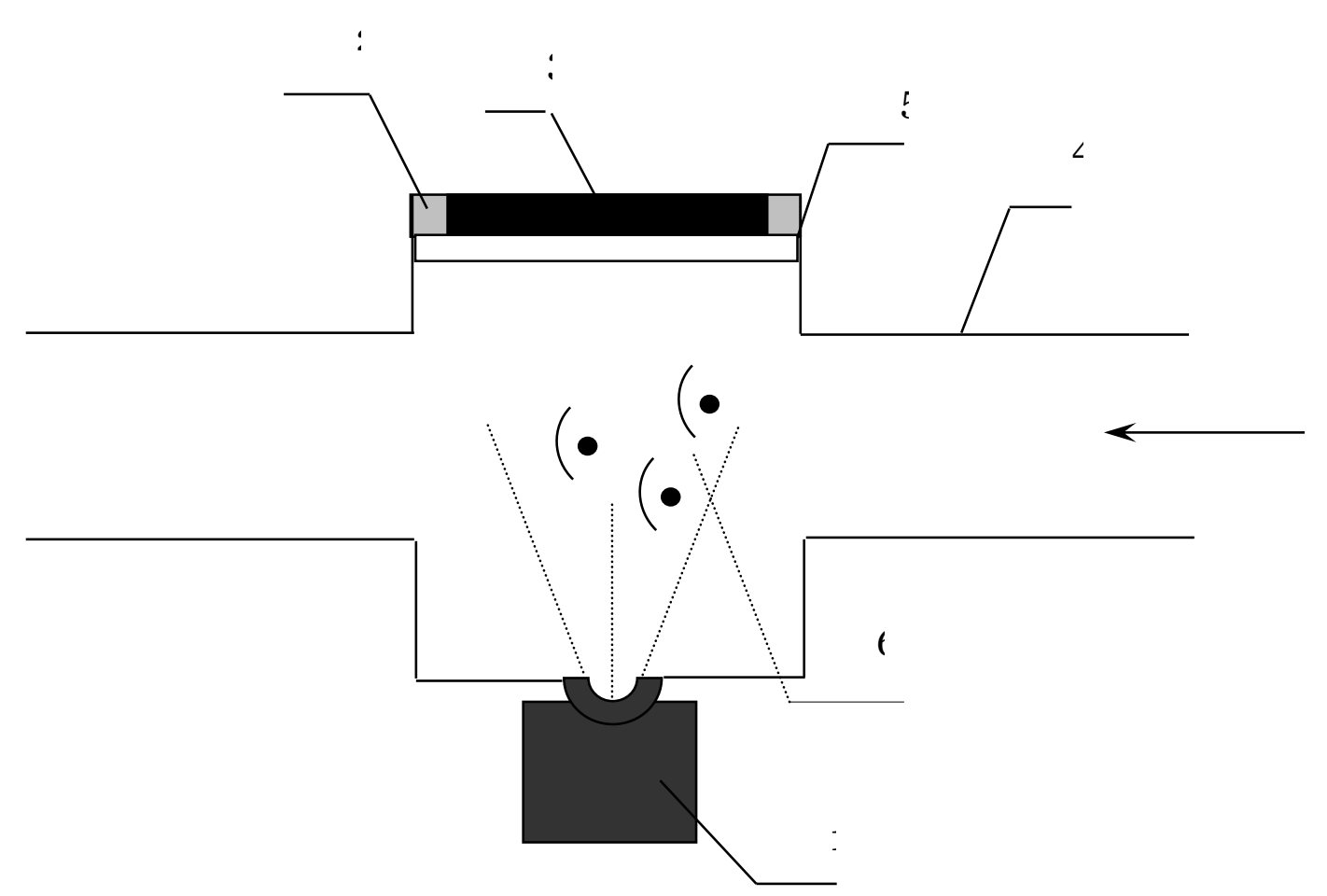

Fig. 3 - Diagram of the shadow recorder.

\section{Setting up experiments}

To solve these problems, a test procedure was developed, focused on the use of a 23millimeter light-gas gun (LGG) as a booster device, which provides the possibility of throwing a particle with the size of $1,4^{*} 0,8^{*} 0,9 \mathrm{~mm}$ to $1,4^{*} 0,8^{*} 1,1 \mathrm{~mm}$ at a speed of $3 \mathrm{~km} / \mathrm{s}$. Since the metal particles have a different size and mass, when leaving the barrel channel and during paraffin evaporation process (Fig.4) particles fly in a "cloud." Therefore, the optical registration scheme should have a sufficiently large registration area $(0.5 \mathrm{~m})$ and a high resolution to visualize the particles themselves and the gas-dynamic pattern accompanying the supersonic motion.

The main difficulty of shadow photo recording was that it was required to carry out optical diagnostics of the state of the object being thrown at $3 \mathrm{~m}$ from the LGG barrel cut. After the thrown object leavies the channel of the, its further free flight occurs together with the products of a ballistic shot (an air - helium mixture with a temperature of about $2000 \mathrm{~K}$ ), due to the small mass of particles ( $0.5 \mathrm{mg}$ to $20 \mathrm{mg}$ ). 


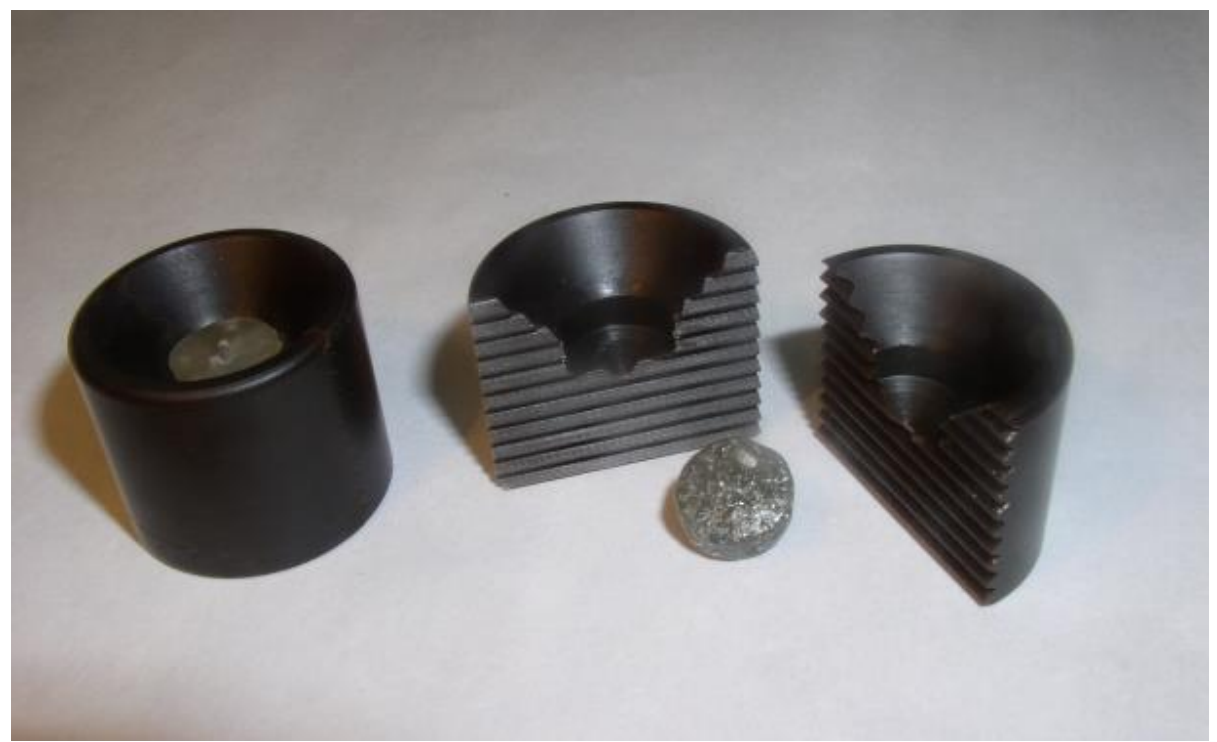

Fig. 4 - tablet of Paraffin, metal particles and leading pallet in assembled and disassembled conditions

The scheme of equipment placement on the working field together with LGG is shown in Fig. 5 .

The moment of starting the light source is the moment of penetration of the film contact sensor installed at the exit of the stand by solid particles which have the lowest ballistic coefficient and, as a consequence, fly in front.

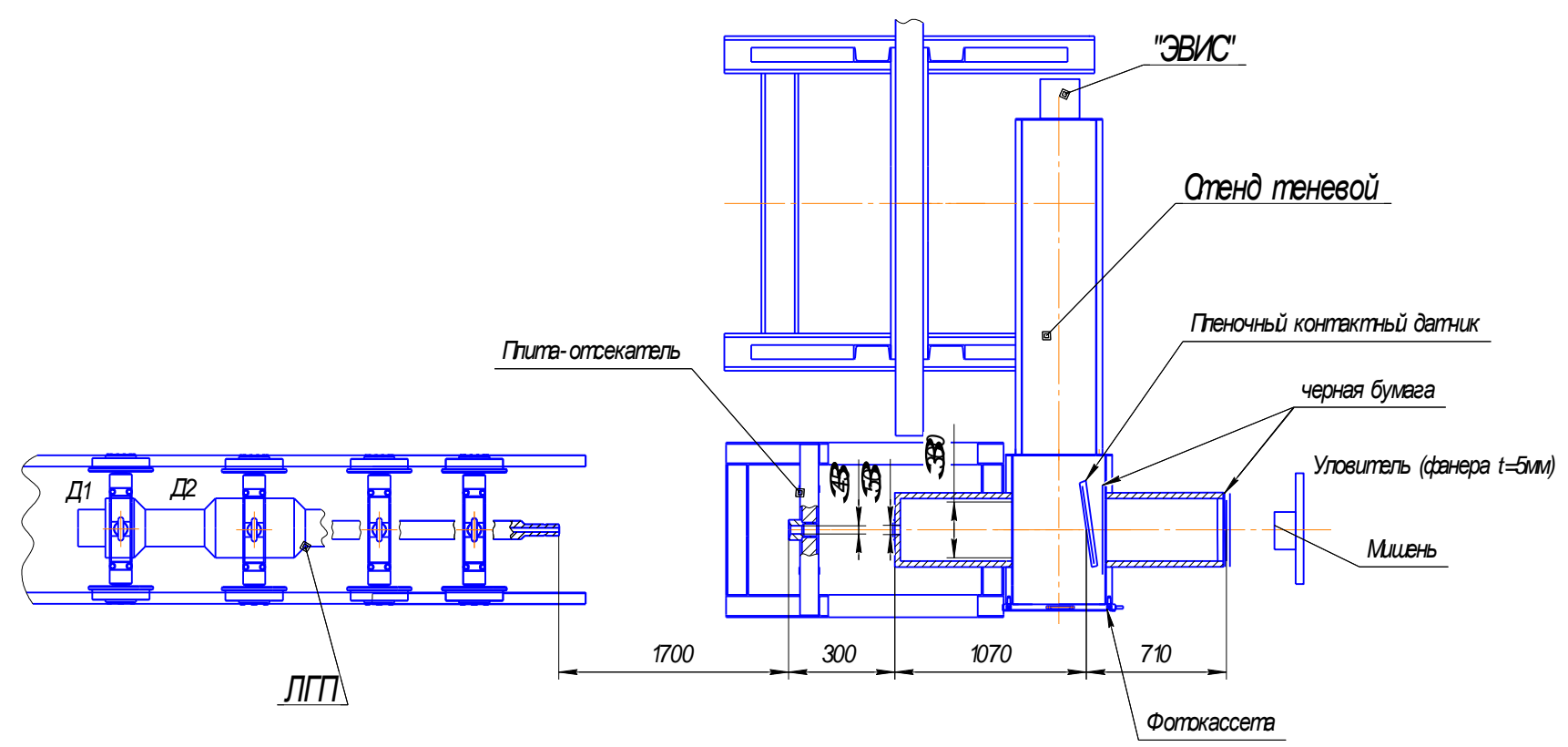

Fig. 5-Layout of the equipment.

As a result of the test experiments, shadow spectra of the flow of solid metal particles were obtained,. Their example is shown in figure 6. 


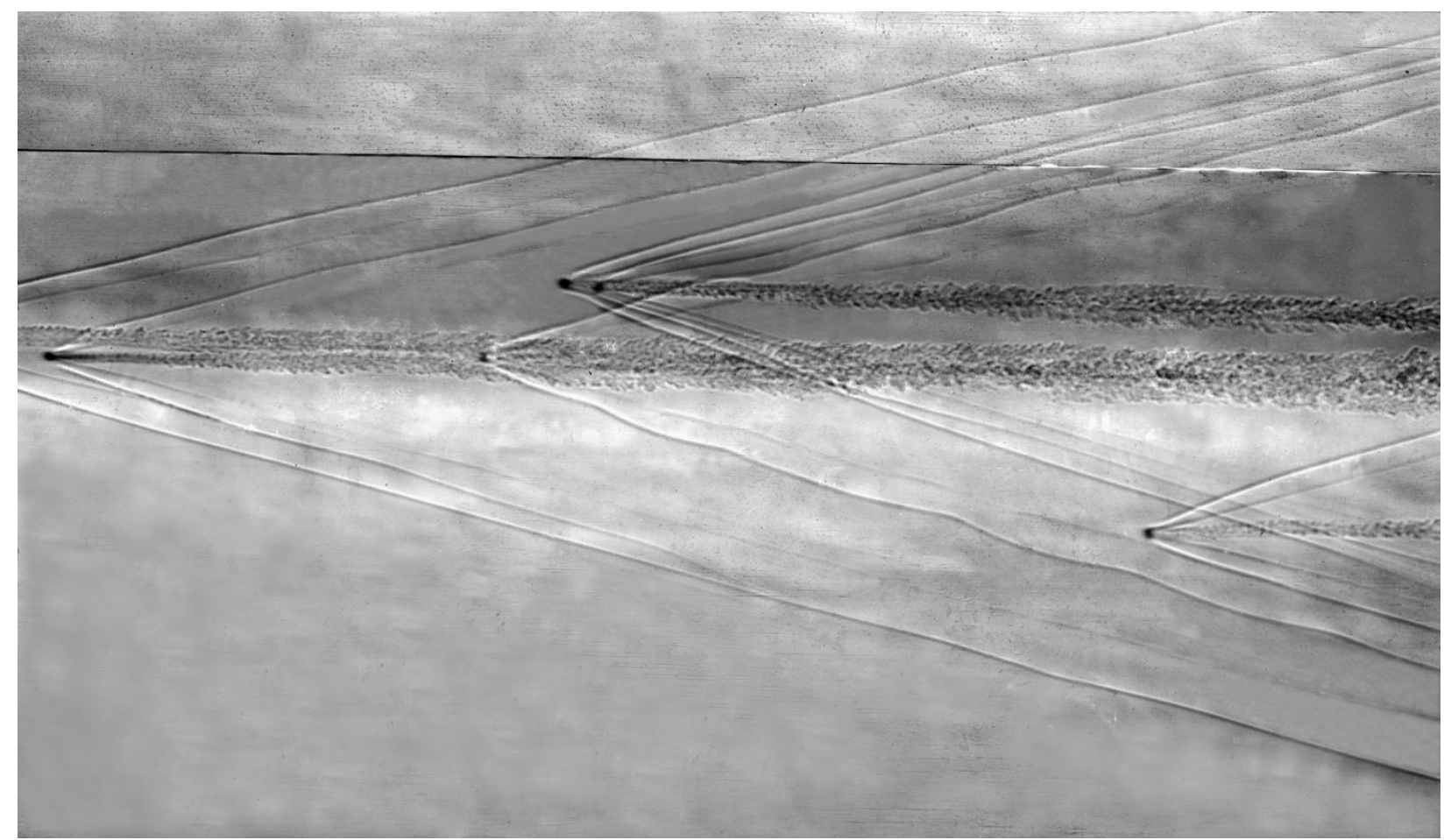

Fig. 6 - Shadow the range of flow of metal particles.

\section{Results analysis}

Through the analysis of negatives, the Mach numbers and the fall of the Mach number of the registered particles on the trajectory section from the LGG trunk to the center of the registration zone in the shadow stand were estimated. The ranges of temperature changes at the breaking point, according to the results of shadow registration, during their flight in the same section of the trajectory were determined.

The shadow flow spectra obtained in the course of experiments are an excellent material for verification of numerical simulation methods of high-speed motion processes of metal particles in gas. In particular, it is possible to study the flow around a group of particles of different diameters under the influence of supersonic air flow. Numerical simulation of the process of flow around solid particles of the material using SolidWorks Flow Simulation [15] is performed. Three-dimensional calculation of the process of external flow around the supersonic flow of the compressible gas was carried out taking into account the relevant boundary conditions, at the entrance to the calculated domains the boundary conditions for the velocity, the condition of adhesion of the flow - on the surface of the particles were set. The complete, Reynolds-averaged Navier-Stokes equations supplemented by a two-parameter turbulence model were solved.

Several calculated cases corresponding to the flight of bodies of approximately the same size and significantly different in particle diameter were considered.

Finite-volume mesh consisted of hexagonal elements, oriented with respect to the direction of flow of the gas at the inlet to the computational domain. In areas of the highest gradients of the parameters of the gas produced adaptation of the mesh by grinding of computational cells. The total number of cells for the case of the flow of particles of different diameters was about 3.3 million cells, for the flow of particles of the same size- 5.7 million cells. The General view of the finite-volume grid is shown in Fig. 7. 


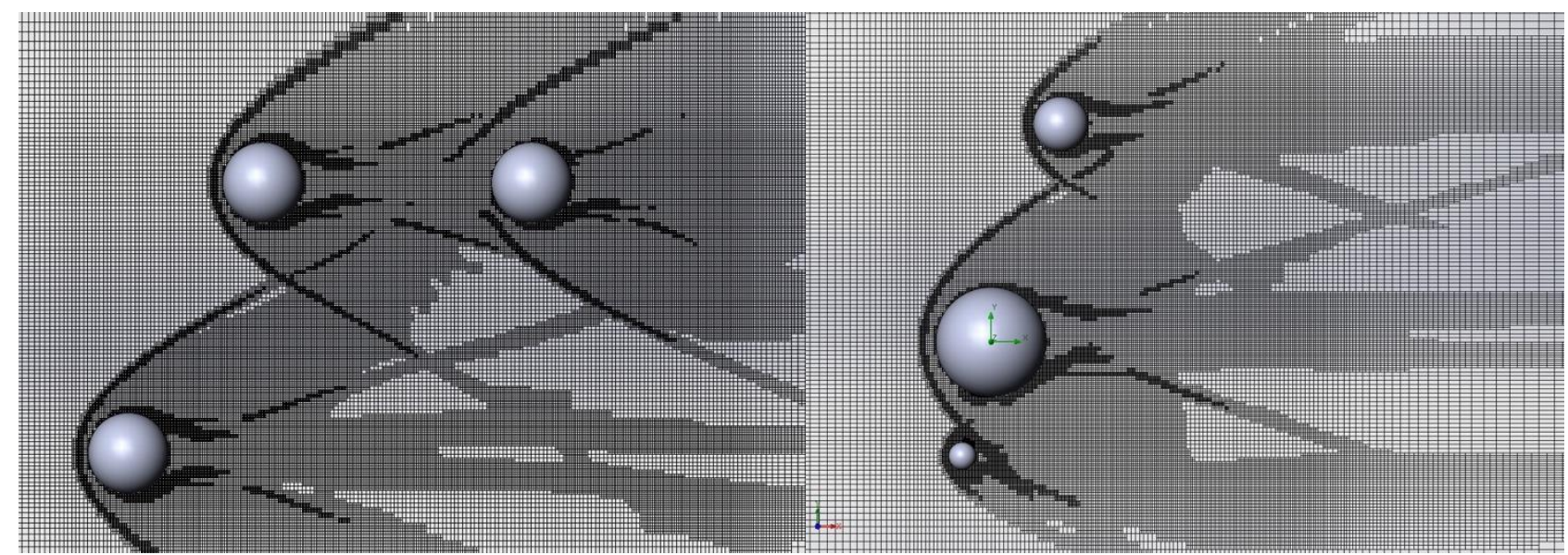

a)

b)

Fig. 7-General view of the adapted finite-volume mesh in the flow simulation around particles with similar sizes (a) and different diameters (b)

Fig. 8.9 shows the distribution patterns of velocity and pressure fields for a group of particles with different and identical sizes in a supersonic gas flow. In general, the flow pattern corresponds to the data recorded in the experiments.

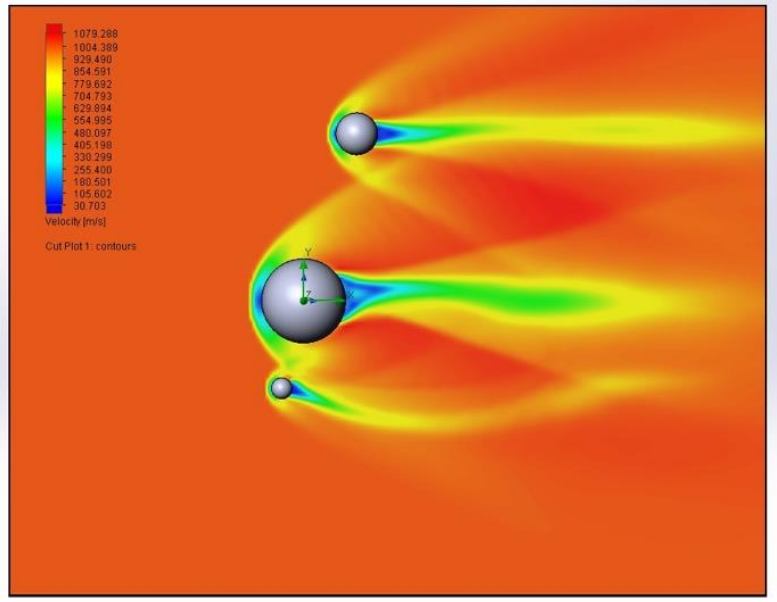

a)

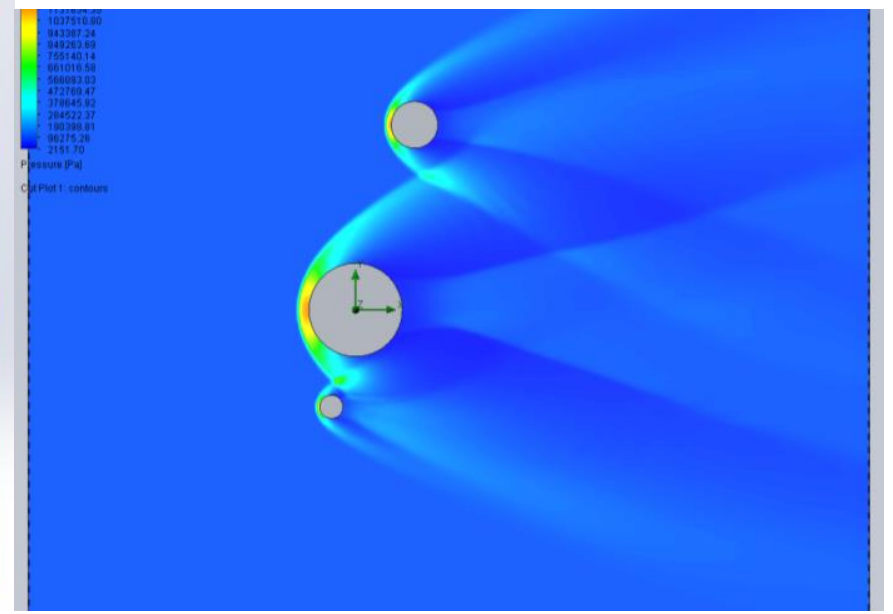

b)

Fig. 8-Distribution of velocity fields (a) and pressure fields (b) in the gas during flow around a group of particles of different sizes 


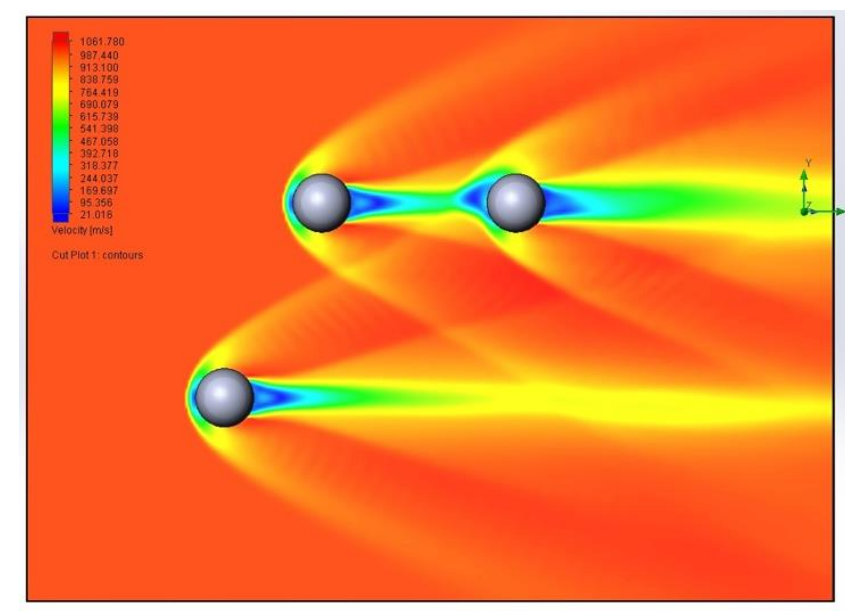

a)

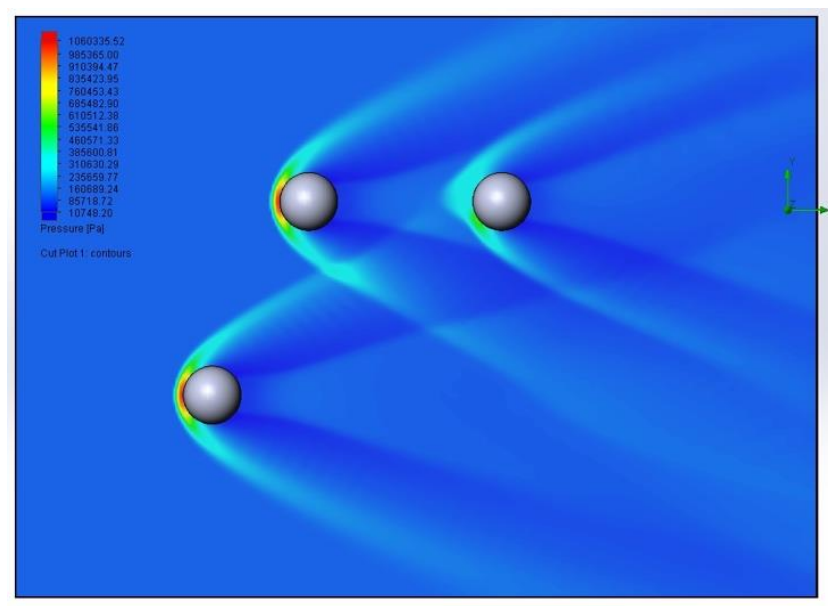

b)

Fig. 9-Distribution of velocity fields (a) and pressure fields (b) in the gas during flow of a group of particles of the same size

According to the experimental data, it was concluded that the flight of metal particles in the air atmosphere with an initial velocity of $\sim 3000 \mathrm{~m} / \mathrm{s}$ at a distance of $\sim 2.9 \mathrm{~m}$ from the cut of the trunk causes intense destruction, melting and evaporation of the particles.

Conclusion

A shadow scheme with a selective range of photorecording was developed. Using this scheme, the shadow spectra of particles simulating the scattering of FM fragments in the event of an accident were obtained.

Analysis of the shadow spectra of the flow around the particles allowed us to determine some quantitative characteristics:

* estimates of the Mach numbers are obtained for the magnitude of the angle of inclination of the shock waves from the particles outside the pattern zone and for two particles in the pattern zone, which were $\mathrm{M}_{1} \approx 4, \mathrm{M} 2 \approx 2.9$ and $\mathrm{M}_{3} \approx 1.5$; based on the fact that the velocities of the registered particles $\mathrm{Vi}=\mathrm{Mi} \times \mathrm{a}$, where $\mathrm{a}=340 \mathrm{~m} / \mathrm{s}$ is the speed of sound in the air, and the calculated value of the initial Mach number is Mlpg $\approx 8.8$, it is possible to estimate the massgeometric characteristics of the particles whose shock waves are visible on the shadow spectrum;

* the sizes of particles in the field of photography are estimated $(\mathrm{R} 1 \approx 0.30 \mathrm{~mm}$-particles flying in front and outside the field of photography; R2 $\approx 0.17 \mathrm{~mm}$-particle flying in front and in the field of photography; $\mathrm{R} 3 \approx 0.11 \mathrm{~mm}$-particle flying behind and in the field of photography). Numerical simulation of the process is carried out.

The work is executed with the support of RFFI grants 17-08-01096, 18-38-00297.

\section{Literature}

1. Gerasimov S. I., Faykov I., Kholin S. A., Cumulative light sources. Sarov: RFNC-VNIIEF, 2002, $143 \mathrm{p}$.

2. Abrukov S. A. Shadow and interference methods of investigation of optical inhomogeneities. Kazan: KSU publishing House, 1962. 81s.

3. Holder D., North R. Shadow methods in aerodynamics. M.:Mir, S. 1966.179

4. Clamex P., Kinghorn M. Development of measurement techniques for hypersonic ballistic units// Technique hypersonic research.M.-L.: Mechanical Engineering, 1964. P. 124-198

5. Merkirch W. Flow Visualization. New York, London: Academic Press, 1974. 250p.

6. Vasiliev L. A. Shadow methods. M.: Science, 1968. 400s 
7. Gerasimov S. I., Chikeev V. A., Lvova E. A., Tatashev K. V., Gerasimov V. R. Spectra of the flow of bodies moving with supersonic speed aerodynamic research. Bulletin of the national research nuclear University MEPhI, 2016, vol. 5, No. 4, pp. 289-293.

8. G. Settles Historical Background-Schlieren and shadowgraph techniques, 2001

9. G. Settles Schlieren and shadowgraph techniques - Visualizing phenomena in transparent media(Book) - Berlin, Germany: Springer-Verlag GmbH, 2001.

10. Dubnishchev, Arbuzov V. A., Belousov p. P. and others. Optical methods of research of streams. Novosibirsk: publishing house of Sibir. UN-TA, 2003. 410s.

11. Belozerov Optical methods of visualization of gas flows. Kazan publishing house. State. Tech. UN-TA, 2007. 747s.

12. Emelyanov V. N., Teterina I. V., Volkov K. N. Visualization of data of physical and mathematical modeling in gas dynamics. [In] 2018, 360s.

13. Gerasimov S. I., V. I. Erofeev, I. I. Kanygin, A. V. Salnikov, R. V. Gerasimov Visualization of muzzle exhaust at a shot from a light-gas gun. Scientific visualization. Vol. 6, №2, 2014.

14. Gerasimov S.I., Erofeev V.I., Kikeev V.A., Fomkin A.P. Various mechanisms of destruction of bodies in hypersonic flow, identified by radiographic registration. Scientific visualization, vol. 7, No. 1, 2015, pp38-44.

15. https://www.solidworks.com/product/solidworks-flow-simulation . 